\title{
Emergency Department and Out-of-Hospital Emergency System (112-AREU 118) integrated response to Coronavirus Disease 2019 in a Northern Italy centre
}

\author{
Stefano Perlini ${ }^{1}$ (D) Fabrizio Canevari ${ }^{2} \cdot$ Sergio Cortesi ${ }^{2} \cdot$ Vito Sgromo $^{2}$ - Antonella Brancaglione ${ }^{2}$. Enrico Contri ${ }^{2}$. \\ Pietro Pettenazza ${ }^{1}$. Francesco Salinaro ${ }^{1}$ - Francesco Speciale $^{1} \cdot$ Giuseppe Sechi $^{3} \cdot$ Claudio Mare $^{3} \cdot$ Sara Cutti $^{4}$. \\ Viola Novelli ${ }^{4}$. Carlo Marena ${ }^{4}$ Alba Muzzi ${ }^{4} \cdot$ Raffaele Bruno $^{5}$ - Alessandra Palo ${ }^{6}$ on behalf of the COVID19 IRCCS San \\ Matteo Pavia Task Force
}

Received: 27 April 2020 / Accepted: 28 May 2020 / Published online: 8 June 2020

c) Società Italiana di Medicina Interna (SIMI) 2020

\begin{abstract}
Since December 2019, the world has been facing the life-threatening disease, named Coronavirus disease-19 (COVID-19), recognized as a pandemic by the World Health Organization. The response of the Emergency Medicine network, integrating "out-of-hospital" and "hospital" activation, is crucial whenever the health system has to face a medical emergency, being caused by natural or human-derived disasters as well as by a rapidly spreading epidemic outbreak. We here report the Pavia Emergency Medicine network response to the COVID-19 outbreak. The "out-of-hospital" response was analysed in terms of calls, rescues and missions, whereas the "hospital" response was detailed as number of admitted patients and subsequent hospitalisation or discharge. The data in the first 5 weeks of the Covid-19 outbreak (February 21-March 26, 2020) were compared with a reference time window referring to the previous 5 weeks (January 17-February 20, 2020) and with the corresponding historical average data from the previous 5 years (February 21-March 26). Since February 21, 2020, a sudden and sustained increase in the calls to the AREU 112 system was noted $(+440 \%)$. After 5 weeks, the number of calls and missions was still higher as compared to both the reference pre-Covid-19 period $(+48 \%$ and $+10 \%$, respectively) and the historical control $(+53 \%$ and $+22 \%$, respectively). Owing to the overflow from the neighbouring hospitals, which rapidly became overwhelmed and had to temporarily close patient access, the population served by the Pavia system more than doubled (from 547.251 to 1.135 .977 inhabitants, + 108\%). To minimize the possibility of intra-hospital spreading of the infection, a separate "Emergency Department—Infective Disease" was created, which evaluated 1241 patients with suspected infection (38\% of total ED admissions). Out of these 1241 patients, 58.0\% $(n=720)$ were admitted in general wards $(n=629)$ or intensive care unit ( $n=91)$. To allow this massive number of admissions, the hospital reshaped many general ward Units, which became Covid-19 Units (up to 270 beds) and increased the intensive care unit beds from 32 to 60 . In the setting of a long-standing continuing emergency like the present Covid-19 outbreak, the integration, interaction and team work of the "out-of-hospital" and "in-hospital" systems have a pivotal role. The present study reports how the rapid and coordinated reorganization of both might help in facing such a disaster. AREU-112 and the Emergency Department should be ready to finely tune their usual cooperation to respond to a sudden and overwhelming increase in the healthcare needs brought about by a pandemia like the current one. This lesson should shape and reinforce the future.
\end{abstract}

Keywords Covid-19 $\cdot$ SARS-CoV-2 $\cdot$ Emergency medical system $\cdot$ Emergency department $\cdot$ Emergency medical system organization

The members of the COVID19 IRCCS San Matteo Pavia Task Force are listed in the Acknowledgements section.

Stefano Perlini

stefano.perlini@unipv.it

Extended author information available on the last page of the article

\section{Introduction}

Since December 2019, the world has been facing the lifethreatening disease, named Coronavirus disease-19 (Covid19), caused by the Severe Acute Respiratory Syndrome Coronavirus-2 (SARS-CoV-2). At first, this epidemic has 
been recognized in Wuhan, China, to subsequently expand and spread throughout the world as a pandemic disease [1]. As of February 29 2020, nearly 80,000 confirmed cases were reported from China [2] and the number of cases in other countries outside China is rapidly increasing, starting from Europe [3] and then spreading almost anywhere in the world. The first indigenous case of COVID 19 in Italy was confirmed on February 21, 2020 in Pavia, arriving from the Codogno Hospital in the Lodi province, i.e. at a $46 \mathrm{~km}$ distance.

According to the April 14, 2020 update on Covid-19 Global Cases by John Hopkins CSSE [4], Italy is only preceded by US and Spain in the number of infected patients, with 159,516 confirmed cases and 20,465 deaths. San Matteo Hospital in Pavia is one to the major referral centres in the epicentre of the Italian epidemic. Up to April 8, out of 1730 patients admitted in the Emergency Department (ED) for suspected Covid-19 infection, 833 were hospitalized. Global hospital mortality accounted for a total of 203 deaths (24.4\%) with a median survival of 4.5 days (IQR 2-9 days). The response of the Emergency Medicine network, integrating "out-of-hospital" and "hospital" activation, is crucial whenever the health system has to face a medical emergency. This does not only apply to natural or human-derived disasters (such as earthquake, tsunami, explosions, train disasters, airplane crash, terrorist attacks, ...) but also to a rapidly spreading epidemic outbreak, such as the present one. In general, a pandemic may be considered a disaster, which is defined as a serious disruption occurring over a short or long period of time that causes widespread human, material, economic or environmental loss that exceeds the ability of the affected community or society to cope using its own resources [5, 6]. A close interaction between the internal and external triage strategies and between the related human and structural resources is a key factor in shaping and morphing the health system preparedness to such events. The present paper reports the Pavia Emergency Medicine network response, showing the main results in the first 5 weeks of the Covid-19 outbreak (February 21-March 26, 2020).

\section{Methods}

\section{The response of the Pavia Emergency Medicine Network to the outbreak}

In our region, the "out-of-hospital" response to any medical emergency is coordinated by the AREU-112 system (Regional Agency of Emergency and Urgency), through the Regional Emergency Urgency Dispatch Centres (SOREU). In detail, the SOREU of the Pianura, in its headquarters in Pavia, handled the first Italian COVID emergency as it manages the territory of the provinces of Pavia, Lodi, Cremona and Mantova. The Dispatch Centre of the Pianura is continuously interfaced with all the hospitals of its territorial reference and in close contact with the Emergency Department of the San Matteo Hospital. In the present situation, characterized by a sudden a sustained emergency due to the Covid-19 outbreak, such an integration is even more important than in usual circumstances. Indeed, the integration between the triage algorithms of the "out-of-hospital" and "hospital" system components has been taking place since February 22, 2020, when the Crisis Unit started a close day-by-day interaction to guide the necessary hospital reshaping. The telephone response and the dispatch triage have been continuously modulated on the type of requests of the population and on hospital and local health protection agency resources that changed in a rapid and difficult to predict manner. This led to a total of 22 revisions of the triage algorithm in only 5 weeks. The four major changes of these revisions are schematically reported in Fig. 1.

On February 23, we separated the access to the ED by diverting the suspected patients to the Infective Disease Outpatient Clinic, which subsequently became the "Emergency Department-Infective Disease". Moreover, in close connection with the structural modifications taking place in the whole hospital [7], we adapted the hospital triage and diagnostic protocols that were shared between the ED and the Infective Disease and Intensive Care Units to optimize patients' care. In the same line, the indications for the diagnostic nasopharyngeal swab and for the immune response assessment were shared with the Virology Unit. The outbreak caused a rapid and sustained increase in the emergency requests from the "out-of-hospital system", associated with an overflow from the neighbouring hospitals (i.e. Lodi, Codogno and Cremona) which rapidly became overwhelmed. Indeed, these hospitals in several occasions asked for a temporary limitation of access (or even a complete closure) due to the overwhelming patients' flow which often exceeded the hospital resources. This caused a sudden increase in calls, ambulance transportations and access to the Pavia ED. Moreover, an increased number of self-presentations posed an additional burden to the healthcare system.

Here we report the Pavia Emergency Department and Out-of-Hospital Emergency System (112-AREU 118) integrated response during the first 5 weeks of the Covid-19 outbreak, as compared to a reference time window referring to the previous 5 weeks, in terms of the different challenges faced by the health system. A further comparison was made by reporting historical data from the same 5 weeks of the previous 5 years.

\section{EMS and ED data}

The data on phone calls, EMS missions, ED admissions with either ambulance transportation or patient 

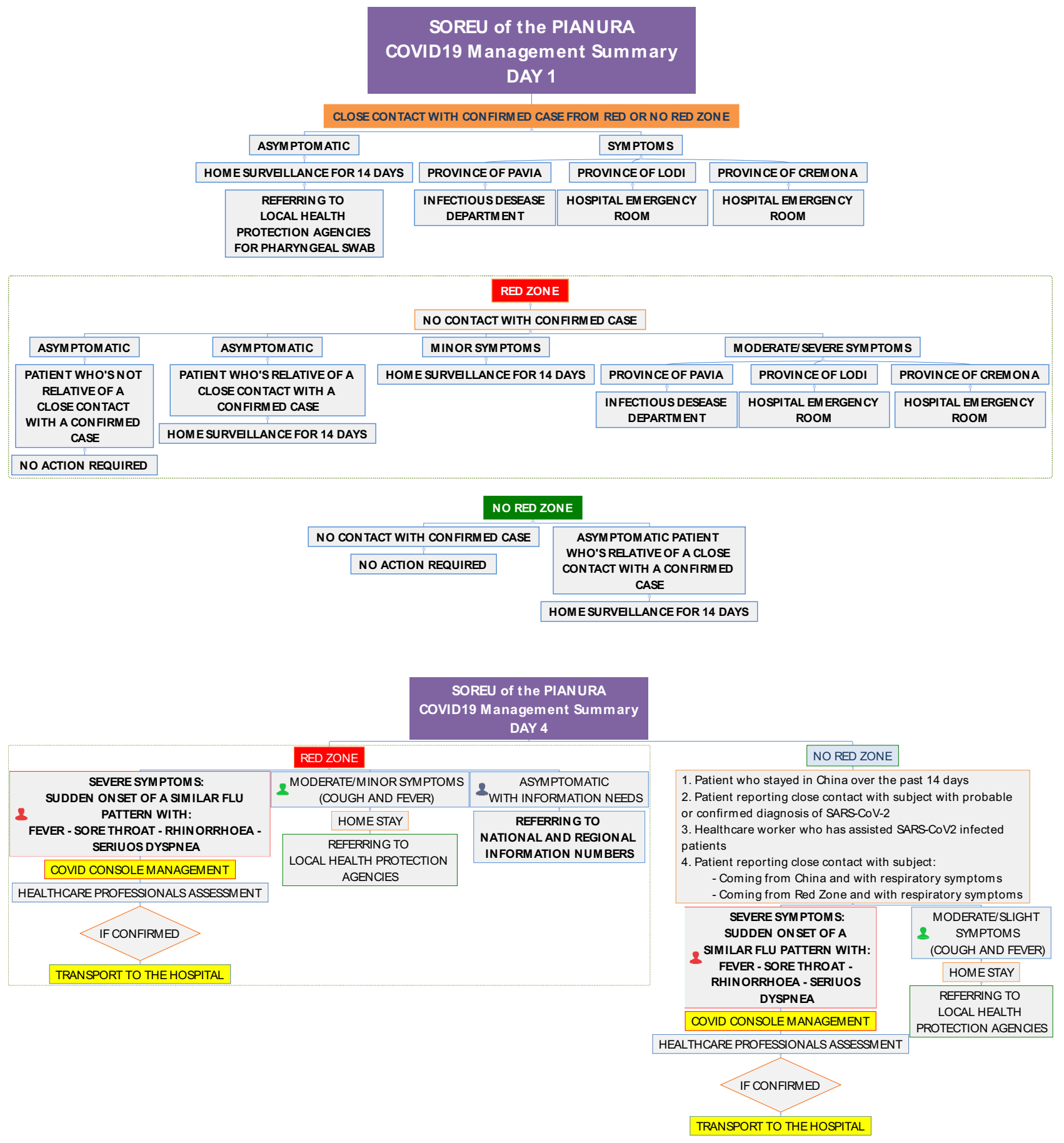

Fig. 1 Schematic representation of the four major changes in the EMS the triage algorithm, taking place during the first 5 weeks of Covid-19 outbreak

self-presentation were stratified per residence area, to assess the local internal (i.e. from the Pavia reference area) as opposed to the external patient burden. As to the hospital response, the number of ED admissions was subdivided between those related or unrelated to Covid-19 suspicion and the further ED decisions were reported in terms of ED discharge or hospital admissions to general wards and intensive care units. Hospital mortality was also recorded. The data collected during the first 5 weeks of the Covid-19 outbreak (February 21-March 26, 2020) were compared with the pre-Covid-195-week reference period (January 17-February 20, 2020) and with the corresponding historical average data from the previous 5 years (February 21-March 26). 

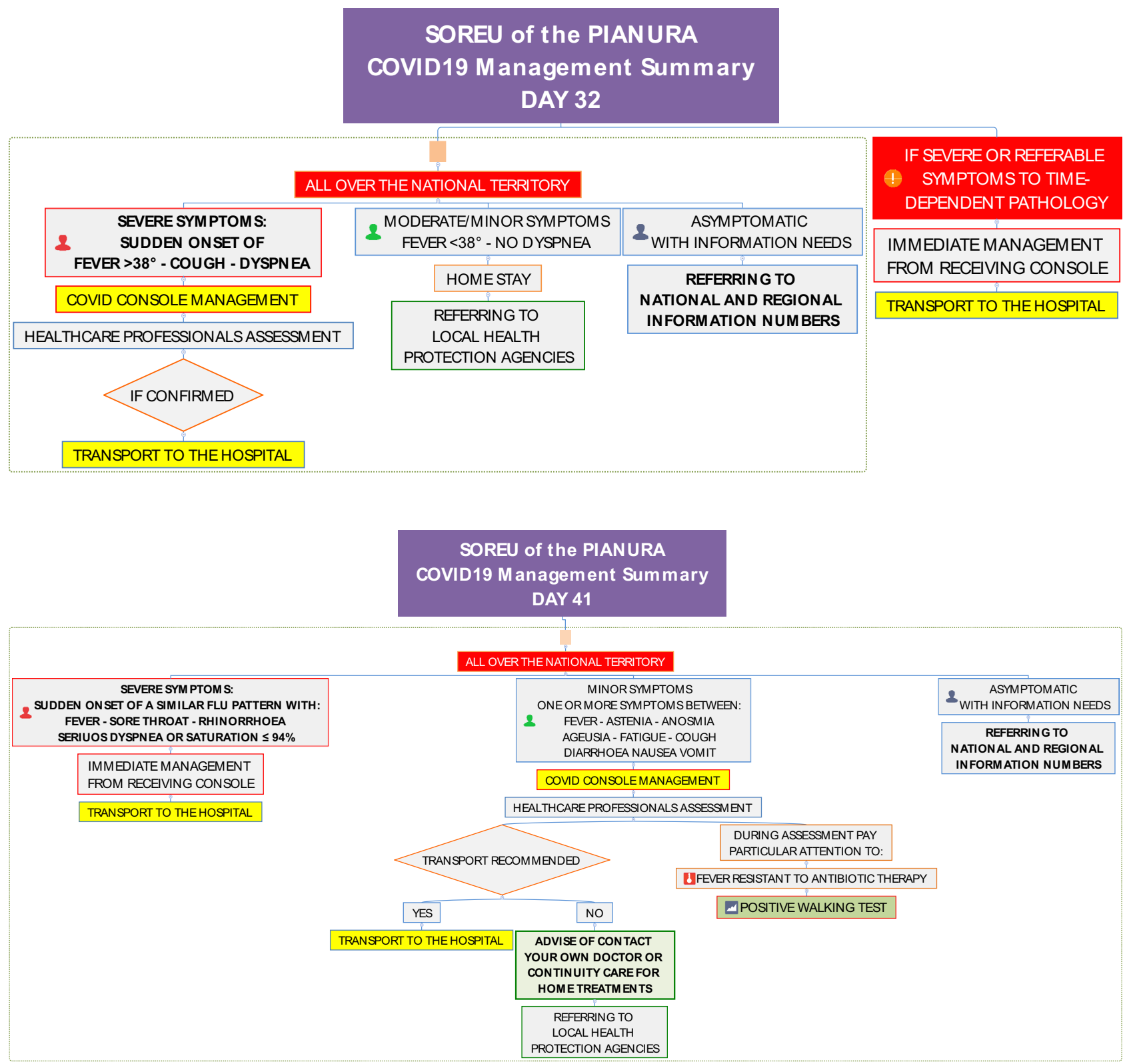

Fig. 1 (continued)

\section{Results}

Since February 21, 2020, a sudden and sustained increase in the calls to the AREU 112 system was noted (reaching up to $+440 \%$ ). A threefold increase in the number of calls was observed in the first week (10904), that in the following 4 weeks set around a mean value of 7794 calls for week. In the 5th week, the number of calls and missions was still higher as compared to both the reference pre-Covid-19 period (January 17-February 20, 2020: $+48 \%$ and $+10 \%$, respectively) and the historical control (February 20-March 26 of the previous 5 years: $+53 \%$ and $+22 \%$, respectively) (Fig. 2).
Up to March 26, the "Emergency Department - Infective Disease" evaluated 1241 patients with suspected SARSCoV-2 infection (38\% of total ED admissions), either from the AREU-112 system ( $n=894,72.0 \%)$ or self-presenting, i.e. patients spontaneously walking in $(n=347,28.0 \%)$. Based on the above-described triage procedures and to the duplication of the Emergency Departments, these patients were separated from patients without the suspicion of SARS-CoV-2 infection directly from the territory (via the "out-of-hospital" triage pathway), to minimize the possibility of intra-hospital spreading of the infection (Fig. 3).

As evident in Fig. 4, an evident shift between Lodi, Codogno and Cremona outbreak areas towards the Pavia 


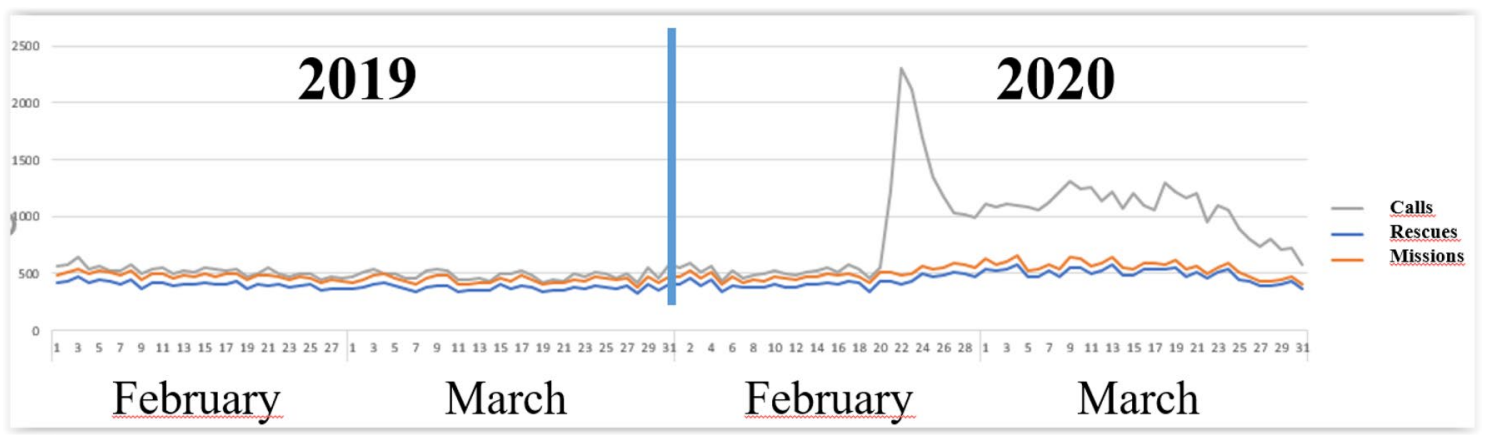

Fig. 2 Calls, rescues and missions according to the AREU 112 records, comparing the different time frames as reported in the "Methods"

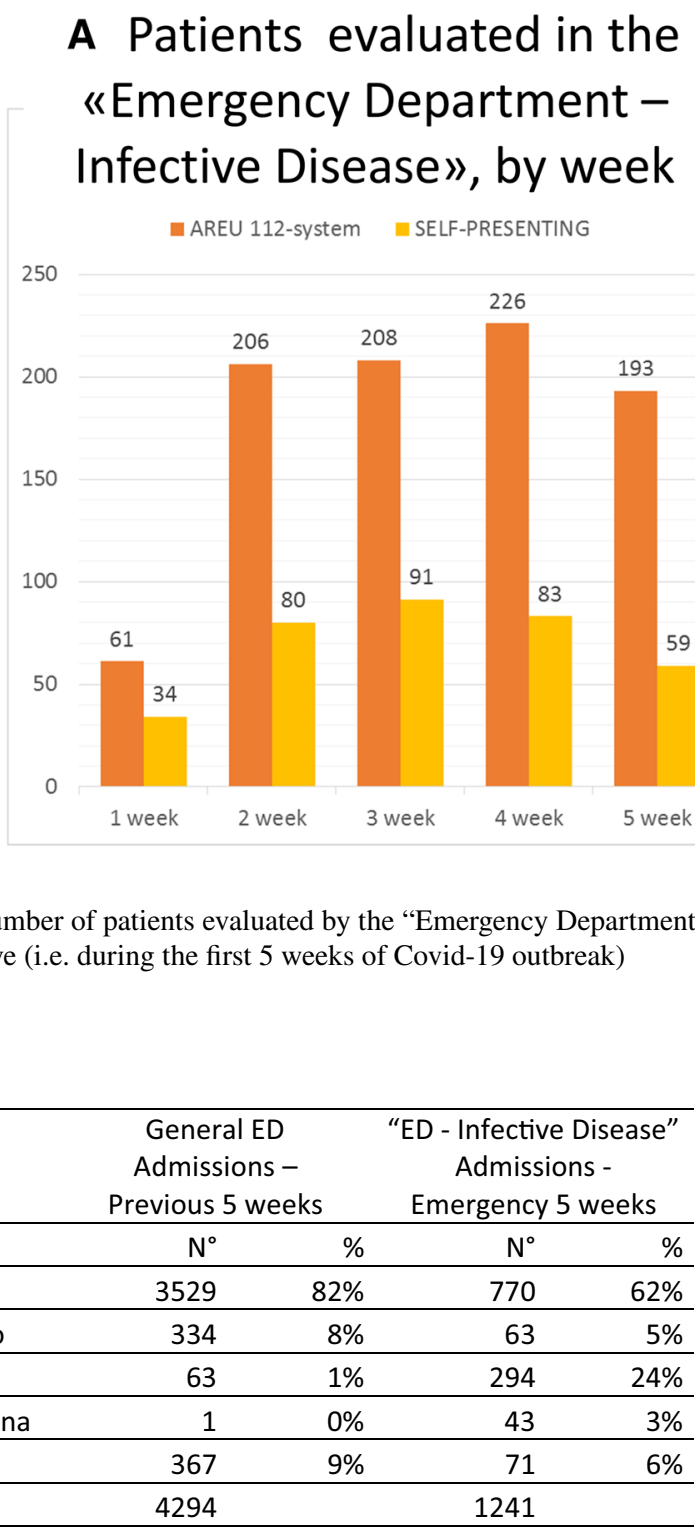

Fig. 4 Timeline evolution of the admissions rate from the outbreak cumulative (i.e. during the first 5 weeks of Covid-19 outbreak) areas

\begin{tabular}{lrrrr}
\hline & $\begin{array}{c}\text { General ED } \\
\text { Admissions - } \\
\text { Previous 5 weeks }\end{array}$ & $\begin{array}{c}\text { "ED - Infective Disease" } \\
\text { Admissions - } \\
\text { Emergency 5 weeks }\end{array}$ \\
\hline Areas & $\mathrm{N}^{\circ}$ & $\%$ & $\mathrm{~N}^{\circ}$ & $\%$ \\
\hline Pavia & 3529 & $82 \%$ & 770 & $62 \%$ \\
\hline Milano & 334 & $8 \%$ & 63 & $5 \%$ \\
\hline Lodi & 63 & $1 \%$ & 294 & $24 \%$ \\
\hline Cremona & 1 & $0 \%$ & 43 & $3 \%$ \\
\hline Other & 367 & $9 \%$ & 71 & $6 \%$ \\
\hline Total & 4294 & & 1241 & \\
\hline
\end{tabular}

\section{B Patients evaluated in the «Emergency Department - Infective Disease», cumulative}

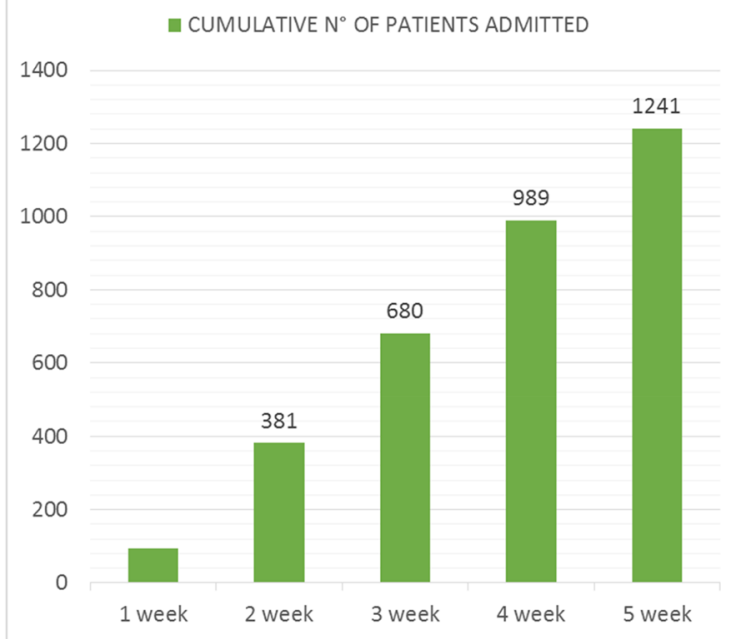

Fig. 3 Number of patients evaluated by the "Emergency Department-Infective Disease" between February 20 and March 26, 2020 by week and

system was noted. Indeed, the admission rate from Lodi, Codogno and Cremona areas that was irrelevant in the past (1\%), accounted for $27 \%$ of total admissions in 5 weeks from February $21(n=337 / 1241)$, peaking at $39 \%$ during the second week $(n=112 / 286)$.

These data underscore the enlargement of the reference area of the Pavia health system from the "usual" areas, mainly confined in the Pavia area, due to the overload of the Lodi, Codogno and Cremona hospitals, which were overwhelmed by the epidemic. To put these data into the perspective of the local population, Pavia area accounts for 547251 inhabitants, whereas Lodi area (including Codogno) 


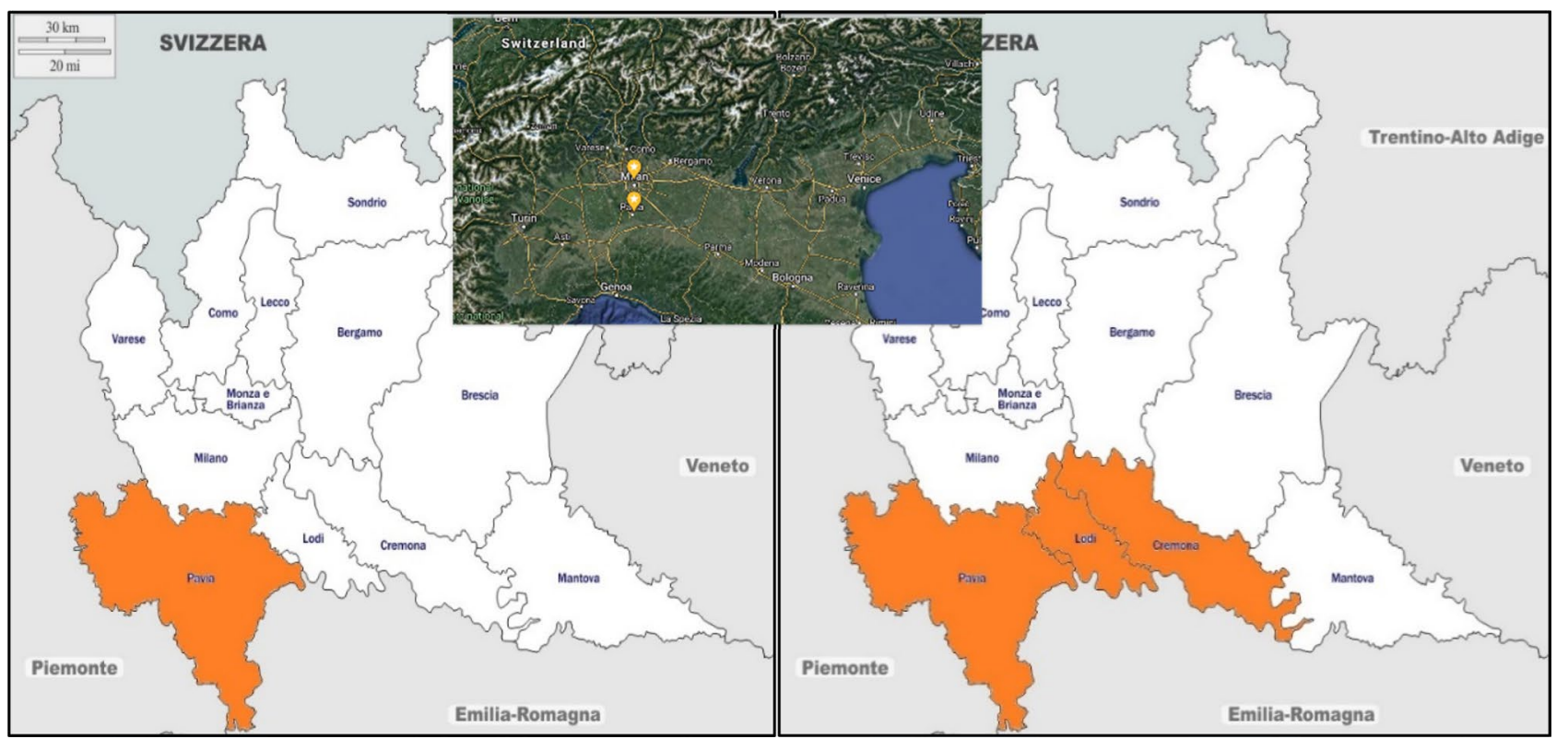

Fig. 5 Left panel: Pavia area $=547251$ inhabitants. Right panel: Pavia + Lodi + Cremona areas $=547251+229338+359388$ inhabitants $($ total $=1135977$, i.e. $+108 \%)$

and Cremona area account for 229338 and 359388 inhabitants, respectively (www.comuni-italiani.it/provincep.html) (Fig. 5).

Out of the 1241 patients evaluated in the "Emergency Department-Infective Disease", 58.0\% $(n=720)$ were admitted in general wards $(n=629)$ or intensive care unit $(n=91)$, according to the clinical needs and the severity of respiratory insufficiency. To allow this massive number of admissions, the hospital reshaped many general ward units, which became Covid-19 Units (up to 270 beds) [8]. In concomitance, intensive care unit beds were almost doubled (from 32 to 60) [9]. Non-invasive ventilation (and C-PAP) was managed both in the Emergency Department and in the Internal Medicine wards, quickly becoming subintensive treatment areas, whereas orotracheal intubation was restricted to the intensive care units. The percentage of patients managed with helmet in general wards rose up to $20 \%(n=126 / 629)$. Median population age (IQR) was 66 years (54-74) in patients admitted in the Internal Medicine wards and 59 years (50-67) in those needing admission to the ICU.

The vast majority of the hospitalized patients $(82.1 \%)$ did enter the ED through the AREU-112 system (+20\% when compared with the previous 5 weeks). Also, the rate of hospitalization for the self-presenting patients (129/347) was higher than in the previous 5 weeks (37.2\% vs. $12.7 \%$ ). A concomitant decrease in "non-COVID" admissions was observed, from 4403 in the 5 weeks before February 21 to 2024 in the reference first 5 weeks of Covid-19 outbreak (i.e. a $54 \%$ decrease). For reference, in the time frame period
"February 21-March 26" of the previous 5 years, 4492 admissions were reported.

\section{Discussion}

The study describes the response of the integrated AREU112-San Matteo Emergency Department system to the Covid-19 outbreak. To face this long-lasting "emergency" situation, re-enforcement and further tightening of the usual collaboration with the AREU-112 have been fundamental. In general, to limit early exposure to the outbreak, triage should aim at identifying and cohorting symptomatic patients [10]. To improve the capability of achieving this goal, a dedicated triage protocol was shared and finely tuned on a dayto-day basis according to the epidemiology of the infection, the request from the territory and the hospital receptivity. Meanwhile, the Emergency Department patient admission pathway was divided in a "clean" and a "dirty" area, by creating an "Emergency Department-Infective Disease" in the Infective Disease building; that is, physically separated from the General Emergency Department [8]. In-hospital triage and diagnostic evaluation protocols were shared between the Emergency Department, the Infective Disease and Intensive Care Units and the Virology Unit, to optimize patients' care. In the "Emergency Department-Infective Disease" triage and diagnostic work-up included: clinical evaluation, Covid-19 blood test panel, hemogasanalysis, chest X-ray, lung ultrasound and electrocardiogram. These data, particularly lung ultrasound, allowed to define a pneumonia pattern 
and to start treatment as soon as positivity of the swab did confirm the diagnosis [11].

Moreover, the patient was not moved from the "Emergency Department-Infective Disease" until the decision discharge/admission was taken, to minimize the risk of infection spreading within the hospital. Sub-intensive treatment areas have been developed to allow non-invasive ventilation both in the Emergency Department and in the General Wards. It has to be noted that during the epidemic, Internal Medicine wards raised considerably the intensity care level. As to the general hospital organization, human and technical resources were activated and strengthened, to enlarge the access routes and the clinical assessment of the patient area in the ED. This allowed to manage a steady improvement in the healthcare staff turnover and a higher quality of care. In the meanwhile, all healthcare providers were given the appropriate personal protection devices [12]. As a further result of this approach, after 5 weeks, we had a prevalence of infected healthcare personnel $<5 \%$. As stated in a recent position paper by the European Society of Emergency Medicine [13], Emergency medical systems, i.e. the front line of the health systems towards the territory, are clearly suffering from overload and severe working conditions, with the additional burden of high risk of contagion and transmission of the health professionals.

Clinical care of patients with suspected Covid-19 infection should focus on early recognition and immediate isolation [10]. Since EDs are concomitantly crowded with patients seeking care for other illnesses [13], the first effort in facing a pandemics like the present one should be in the direction of a clear separation of patient pathways, in order to prevent a further spread of the infection [14]. Indeed, beyond the clinical responsibility of early triage and management of patients with suspected or confirmed infection, Emergency workers have a crucial role in preventing infection spreading. One example reported that during the MERS-CoV outbreak in an Emergency Department in Singapore 82 individuals (including healthcare workers) were infected by a single case [15]. This is the main reason guiding the need of a clear separation between patients' track starting from the "out-of-hospital" Emergency System. Since the first positive cases for SARS-CoV-2 infection in the Lodi area on February 21, Pavia health system has started a process of rapid and continuous adaptation and morphing to face this new emergency, both in the "out-of-hospital" emergency system and in the "in-hospital" management. The "out-of-hospital" emergency system, headed by AREU-112 has adapted the response model to the impressive number of requests for help and information regarding the new infection. Since the beginning of the Covid-19 outbreak, AREU-112 increased and strengthened the Unique Response Centres and activated new dedicated Public Safety Answering Points, both at the regional and at the local level. In particular, the Regional
Emergency Urgency Dispatch Centre (SOREU) of the Pianura, which responds to requests for medical assistance for the provinces of Pavia, Lodi, Cremona and Mantova, was immediately reorganized by activating an additional response console and a dedicated telephone triage path for patients potentially affected by Covid-19, in order to allocate them to the most suitable hospitals and to evaluate the possibility of home management. In a first phase of the disaster, the SOREU of the Pianura took advantage of the support of two resident figures. An Infectious Disease Resident supported the health dispatch section of the SOREU in selected cases and an Emergency Medicine Resident was a further and continuous connection with the Emergency RoomInfective Disease of San Matteo Hospital, with the aim of optimizing patient flow to the different hospital structures. Impressive have been the numbers: since the beginning of the emergency, SOREU of the Pianura has managed more than 1700 patients with symptoms compatible with the new SARS-CoV-2 infection, has processed up to 2300 calls/ day as compared to the ordinary 500 and has increased the ambulances in the area of several dozens, as well as having fielded numerous special ambulances for the exclusive transport of COVID patients, some of them with qualified nurses for the emergency, in addition to rapid response medical team in equivalent numbers in the ordinary situation.

Another important point was the decrease in nonCovid-19 ED admissions when compared with the previous 5 weeks as well as with the previous 5 years (2204 vs. 4403 and 4492 admissions, respectively). Such an important decrease in non-Covid-19 admissions during the outbreak was caused by several reasons, among which the patient's fear to enter the hospital risking a possible contact with infected patients took a relevant role. The likely consequences in terms of missed/delayed diagnoses are beyond the scope of the present study, but are indeed one of the major clinical consequences of Covid-19 pandemics, adding on the devastating effects on infected patients. This point has been underscored by several reports, one example being represented by the reduced admission rate for acute coronary syndrome [16] with the concomitant increase in out-of-hospital cardiac arrest [17].

Healthcare organization in the emergency and critical care setting is crucially dependent on the close interaction and team work of the "out-of-hospital" and "in-hospital" systems and the present study reports how the rapid and coordinated reorganization of both is fundamental. The fine tuning of the "out-of-hospital" and "in-hospital" triage protocols has to be rearranged on a day-to-day basis according to the epidemiology of the infection, the request from the territory and the hospital receptivity. Indeed, the preparedness of the Emergency Departments for responding to any emergency or disaster is crucial and the close link between the hospital Emergency Department and the 
"out-of-hospital" system is confirmed as one of the key factors in the response to any disaster. The disaster scenario is characterized by a sudden and overwhelming overload of the health system caused by natural or human-derived disasters, which is dramatic yet limited in a relatively short-lasting time window (hours, days). In contrast, a rapidly spreading epidemic outbreak, such as the present one is characterized by a sudden, yet long-lasting disaster (weeks, months). A consequence of such a time frame is the need of a continuous modulation of the response of the Emergency System, with a close interaction between the AREU-112 system and the ED.

\section{Conclusions}

In the setting of a long standing and ongoing disaster like the present Covid-19 outbreak, the integration, interaction and team work of the "out-of-hospital" and "in-hospital" systems have a pivotal role. The present study reports how the rapid and coordinated reorganization of both might help in facing such a disaster. AREU-112 and the Emergency Department should be ready to finely tune their usual cooperation in order to respond to a sudden and overwhelming increase in the healthcare needs brought about by a pandemia like the current one. This lesson should shape and reinforce the future.

Acknowledgements All patients, families, staff personnel and volunteers who are helping us in facing Covid-19 pandemia. Covid-19 IRCCS San Matteo Pavia Task Force: Infective Disease Unit: Staff: Raffaele Bruno, Mario U Mondelli, Enrico Brunetti, Angela Di Matteo, Elena Seminari, Laura Maiocchi, Valentina Zuccaro, Layla Pagnucco, Bianca Mariani, Serena Ludovisi, Raffaella Lissandrin, Aldo Parisi, Paolo Sacchi, Savino FA Patruno, Giuseppe Michelone, Roberto Gulminetti, Domenico Zanaboni, Stefano Novati, Renato Maserati, Paolo Orsolini, Marco Vecchia. Residents: Marco Sciarra, Erika Asperges, Marta Colaneri, Alessandro Di Filippo, Margherita Sambo, Simona Biscarini, Matteo Lupi, Silvia Roda, Teresa Chiara Pieri, Ilaria Gallazzi, Michele Sachs, Pietro Valsecchi. Emergency Care Unit: Staff: Stefano Perlini, Claudia Alfano, Marco Bonzano, Federica Briganti, Giuseppe Crescenzi, Anna Giulia Falchi, Roberta Guarnone, Barbara Guglielmana, Elena Maggi, Ilaria Martino, Pietro Pettenazza, Serena Pioli di Marco, Federica Quaglia, Anna Sabena, Francesco Salinaro, Francesco Speciale, Ilaria Zunino, Niccolò Furlan; Gabriele Savioli. Residents: Marzia De Lorenzo, Gianmarco Secco, Lorenzo Dimitry, Giovanni Cappa, Igor Maisak, Benedetta Chiodi, Massimiliano Sciarrini, Bruno Barcella, Flavia Resta, Luca Moroni, Giulia Vezzoni, Lorenzo Scattaglia, Elisa Boscolo, Caterina Zattera, Tassi Michele Fidel, Capozza Vincenzo, Marco Bazzini, Elena Lago, Federico Bracchi, Giulia Sturniolo. Intensive Care Unit: Giorgio Iotti, Francesco Mojoli, Mirko Belliato, Luciano Perotti, Silvia Mongodi, Guido Tavazzi. Paediatric Unit: Gianluigi Marseglia, Amelia Licari, Ilaria Brambilla. Virology Unit: Staff: Barbarini Daniela, Bruno Antonella, Cambieri Patrizia, Campanini Giulia, Comolli Giuditta, Corbella Marta, Daturi Rossana, Furione Milena, Mariani Bianca, Maserati Roberta, Monzillo Enza, Paolucci Stefania, Parea Maurizio, Percivalle Elena, Piralla Antonio, Rovida Francesca, Sarasini Antonella, Zavattoni Maurizio. Technical staff: Adzasehoun Guy, Bellotti Laura, Cabano Ermanna, Casali Giuliana, Dossena Luca, Frisco Gabriella, Garbagnoli Gabriella, Girello
Alessia, Landini Viviana, Lucchelli Claudia, Maliardi Valentina, Pezzaia Simona, Premoli Marta. Residents: Bonetti Alice, Caneva Giacomo, Cassaniti Irene, Corcione Alfonso, Di Martino Raffella, Di Napoli Annapia, Ferrari Alessandro, Ferrari Guglielmo, Fiorina Loretta, Giardina Federica, Mercato Alessandra, Novazzi Federica, Ratano Giacomo, Rossi Beatrice, Vecchio Nepita Edoardo. Research Laboratories, Division of Infectious Diseases and Immunology: Antonella Cerino, Stefania Varchetta, Barbara Oliviero, Stefania Mantovani, Dalila Mele. Pharmacy Unit: Monica Calvi, Michela Tizzoni. Hospital Management: Carlo Nicora, Antonio Triarico, Vincenzo Petronella, Carlo Marena, Alba Muzzi, Paolo Lago. Data Unit: Marco Rettani, Francesco Comandatore, Gherard Bissignandi, Stefano Gaiarsa, Claudio Bandi, Alessanda Ferrari, Francesca Pasi

Author contributions SP, AP, AM and SC conceived the study. CM, GS and CM supervised the conduct of the trial and data collection. FC, SC, VS, AB, EC, PP, FS, FS, SC, VN, AM, undertook recruitment of participating centres and patients and managed the data, including quality control. SP, AP, SC, RB and AM provided statistical advice on study design and analyzed the data; SP drafted the manuscript and all authors contributed substantially to its revision. SP and AP take responsibility for the paper as a whole.

Funding No commercial interest, financial source or material support to disclose.

\section{Compliance with ethical standards}

Conflict of interest The authors declare that they have no conflict of interest.

Human and animal rights statement The study protocol was approved by the local ethics committees and was conducted in accordance with the Declaration of Helsinki.

Informed consent Written informed consent was collected according to local practice upon patient admission to the Emergency Department.

\section{References}

1. Velavan TP, Meyer CG (2020) The COVID-19 epidemic. Trop Med Int Health 25(3):278-280. https://doi.org/10.1111/tmi.13383

2. Guan WJ, Ni ZY, Hu Y, Liang WH, Ou CQ, He JX, Liu L, Shan H, Lei CL, Hui DSC, Du B, Li LJ, Zeng G, Yuen KY, Chen RC, Tang CL, Wang T, Chen PY, Xiang J, Li SY, Wang JL, Liang ZJ, Peng YX, Wei L, Liu Y, Hu YH, Peng P, Wang JM, Liu JY, Chen Z, Li G, Zheng ZJ, Qiu SQ, Luo J, Ye CJ, Zhu SY, Zhong NS, China Medical Treatment Expert Group for C (2020) Clinical characteristics of coronavirus disease 2019 in China. N Engl J Med. https://doi.org/10.1056/NEJMoa2002032

3. Eurosurveillance Editorial T (2020) Updated rapid risk assessment from ECDC on the novel coronavirus disease 2019 (COVID-19) pandemic: increased transmission in the EU/EEA and the UK. Eurosurveillance. https://doi.org/10.2807/1560-7917. Es.2020.25.10.2003121

4. John Hopkins Coronavirus Resource Center (2020). https://coron avirus.jhu.edu/map.html. Accessed 14 April 2020

5. Nekoie-Moghadam M, Kurland L, Moosazadeh M, Ingrassia PL, Della Corte F, Djalali A (2016) Tools and checklists used for the evaluation of hospital disaster preparedness: a systematic review. Disaster Med Public Health Prep 10(5):781-788. https://doi. org/10.1017/dmp.2016.30 
6. Ingrassia PL, Mangini M, Azzaretto M, Ciaramitaro I, Costa L, Burkle FM Jr, Della Corte F, Djalali A (2016) Hospital disaster preparedness in Italy: a preliminary study utilizing the World Health Organization Hospital Emergency Response Evaluation Toolkit. Minerva Anestesiol 82(12):1259-1266

7. Asperges E, Novati S, Muzzi A, Biscarini S, Sciarra M, Lupi M, Sambo M, Gallazzi I, Peverini M, Lago P, Mojoli F, Perlini S, Bruno R (2020) Rapid response to COVID-19 outbreak in Northern Italy: how to convert a classic infectious disease ward into a COVID-19 response centre. J Hosp Infect. https://doi. org/10.1016/j.jhin.2020.03.020

8. Asperges E, Novati S, Muzzi A, Biscarini S, Sciarra M, Lupi M, Sambo M, Gallazzi I, Peverini M, Lago P, Mojoli F, Perlini S, Bruno R, Force C-ISMPT (2020) Rapid response to COVID-19 outbreak in Northern Italy: how to convert a classic infectious disease ward into a COVID-19 response centre. J Hosp Infect. https://doi.org/10.1016/j.jhin.2020.03.020

9. Mojoli F, Mongodi S, Grugnetti G, Muzzi A, Baldanti F, Bruno R, Triarico A, Antonio Iotti G (2020) Setup of a dedicated coronavirus intensive care unit: logistical aspects. Anesthesiology. https ://doi.org/10.1097/ALN.0000000000003325

10. Whiteside T, Kane E, Aljohani B, Alsamman M, Pourmand A (2020) Redesigning emergency department operations amidst a viral pandemic. Am J Emerg Med. https://doi.org/10.1016/j. ajem.2020.04.032(in press)

11. Soldati G, Smargiassi A, Inchingolo R, Buonsenso D, Perrone T, Briganti DF, Perlini S, Torri E, Mariani A, Mossolani EE, Tursi F, Mento F, Demi L (2020) Is there a role for lung ultrasound during the COVID-19 pandemic? J Ultrasound Med. https://doi. org/10.1002/jum.15284

12. Holland M, Zaloga DJ, Friderici CS (2020) COVID-19 personal protective equipment (PPE) for the emergency physician. Vis J Emerg Med 19:100740. https://doi.org/10.1016/j.visj.2020.10074 0

13. Garcia-Castrillo L, Petrino R, Leach R, Dodt C, Behringer W, Khoury A, Sabbe M (2020) European Society For emergency medicine position paper on emergency medical systems response to COVID-19. Eur J Emerg Med. https://doi.org/10.1097/ MEJ.0000000000000701

14. Wee LE, Fua TP, Chua YY, Ho FWA, Sim XYJ, Conceicao EP, Venkatachalam I, Tan KB, Tan BH (2020) Containing COVID19 in the emergency room: the role of improved case detection and segregation of suspect cases. Acad Emerg Med. https://doi. org/10.1111/acem.13984

15. Cho SY, Kang JM, Ha YE, Park GE, Lee JY, Ko JH, Lee JY, Kim JM, Kang CI, Jo IJ, Ryu JG, Choi JR, Kim S, Huh HJ, Ki CS, Kang ES, Peck KR, Dhong HJ, Song JH, Chung DR, Kim YJ (2016) MERS-CoV outbreak following a single patient exposure in an emergency room in South Korea: an epidemiological outbreak study. Lancet (London, England) 388(10048):994-1001. https://doi.org/10.1016/S0140-6736(16)30623-7

16. De Filippo O, D’Ascenzo F, Angelini F, Bocchino PP, Conrotto F, Saglietto A, Secco GG, Campo G, Gallone G, Verardi R, Gaido L, Iannaccone M, Galvani M, Ugo F, Barbero U, Infantino V, Olivotti L, Mennuni M, Gili S, Infusino F, Vercellino M, Zucchetti O, Casella G, Giammaria M, Boccuzzi G, Tolomeo P, Doronzo B, Senatore G, Grosso Marra W, Rognoni A, Trabattoni D, Franchin L, Borin A, Bruno F, Galluzzo A, Gambino A, Nicolino A, Truffa Giachet A, Sardella G, Fedele F, Monticone S, Montefusco A, Omede P, Pennone M, Patti G, Mancone M, De Ferrari GM (2020) Reduced rate of hospital admissions for ACS during Covid-19 outbreak in Northern Italy. N Engl J Med. https://doi. org/10.1056/NEJMc2009166

17. Baldi E, Sechi GM, Mare C, Canevari F, Brancaglione A, Primi R, Klersy C, Palo A, Contri E, Ronchi V, Beretta G, Reali F, Parogni P, Facchin F, Bua D, Rizzi U, Bussi D, Ruggeri S, Oltrona Visconti L, Savastano S, Lombardia CR (2020) Out-of-hospital cardiac arrest during the Covid-19 outbreak in Italy. N Engl J Med. https://doi.org/10.1056/NEJMc2010418

Publisher's Note Springer Nature remains neutral with regard to jurisdictional claims in published maps and institutional affiliations.

\section{Affiliations}

\section{Stefano Perlini ${ }^{1}$ - Fabrizio Canevari ${ }^{2} \cdot$ Sergio Cortesi ${ }^{2} \cdot$ Vito Sgromo $^{2}$ - Antonella Brancaglione ${ }^{2}$ - Enrico Contri ${ }^{2}$. Pietro Pettenazza ${ }^{1}$. Francesco Salinaro ${ }^{1}$ - Francesco Speciale ${ }^{1}$. Giuseppe Sechi ${ }^{3}$. Claudio Mare $^{3}$ - Sara Cutti ${ }^{4}$. Viola Novelli ${ }^{4}$. Carlo Marena ${ }^{4}$ - Alba Muzzi ${ }^{4}$. Raffaele Bruno ${ }^{5}$. Alessandra Palo ${ }^{6}$ on behalf of the COVID19 IRCCS San Matteo Pavia Task Force}

1 Emergency Medicine Unit and Emergency Medicine Postgraduate Training Program, IRCCS Policlinico San Matteo Foundation, Department of Internal Medicine, University of Pavia, Pavia, Italy

2 SOREU Pianura, AREU (Azienda Regionale Emergenza Urgenza), Pavia, Italy

3 AREU (Azienda Regionale Emergenza Urgenza), Milan, Italy
4 Medical Direction, IRCCS Policlinico San Matteo Foundation, Pavia, Italy

5 Infectious Disease Unit, IRCCS Policlinico San Matteo Foundation, University of Pavia, Pavia, Italy

6 AAT 118 Pavia, AREU (Azienda Regionale Emergenza Urgenza), Department of Intensive Medicine, Policlinico San Matteo Foundation, Pavia, Italy 